\title{
Subsurface disorder and electro-optical properties of proton-exchanged $\mathrm{LiNbO}_{3}$ waveguides produced by different techniques
}

S. M. Kostritskii skostritskii@optolink.ru

\section{Y. N. Korkishko}

\section{A. Fedorov}

\section{P. Mitrokhin}

O. G. Sevostyanov

\section{M. Chirkova}

O. Stepanenko

\section{De Micheli}

RPC Optolink Ltd, 124489, Zelenograd, Russia

RPC Optolink Ltd, 124489, Zelenograd, Russia

RPC Optolink Ltd, 124489, Zelenograd, Russia

RPC Optolink Ltd, 124489, Zelenograd, Russia

Phys. Dept., Kemerovo State University, 650043, Kemerovo, Russia

Phys. Dept., Kemerovo State University, 650043, Kemerovo, Russia

LPMC, University de Nice Sophia-Antipolis, 06100 Nice Cedex, France

Present address : MPQ, Universit Paris Diderot,2, Place Jussieu, 75251 Cedex 05 Paris

LPMC, University de Nice Sophia-Antipolis, 06100 Nice Cedex, France

It has been established, that proton-exchanged LiNb03 waveguides have a marked subsurface layer with structural disorder inducing degradation of electro-optical properties of these waveguides. At the same time, such a subsurface disorder is found to be less pronounced in soft proton-exchanged (SPE) waveguides in comparison with annealed proton-exchanged (APE) ones. The experimental samples of phase modulators fabricated by SPE technique exhibit a better electro-optical efficiency compared to the LiNb03 modulators produced by the standard and improved APE techniques.

[DOI: http://dx.doi.org/10.2971/jeos.2014.14055]

Keywords: Optical waveguides, Lithium Niobate, electro-optic effect, proton exchange

\section{INTRODUCTION}

Annealed proton exchange (APE) is now an established technique for fabricating integrated optical devices in $\mathrm{LiNbO}_{3}$, as it allows obtaining low-loss waveguides with good electrooptical (EO) performances [1]-[4]. Note that a significant degradation (more than one order of magnitude) of the EO coefficients occurs immediately after the proton exchange process [1, 2]. However, after an appropriate annealing in order to obtain $\mathrm{LiNbO} 3$ waveguides in the so-called -phase [2]-[4], the EO coefficients have almost recovered their initial values. Nevertheless, EO coefficients cannot be completely restored even after a long annealing, when the initial proton exchange has been performed using conditions that are in common use today [2]-[4]. It was shown [5] that an extended annealing in dry air at $\mathrm{T} \geq 320^{\circ} \mathrm{C}$, leads to the precipitation of disordered phases in a subsurface layer (depth $\leq 0.5 \mu \mathrm{m}$ ), causing undesirable degradation of the EO properties of the waveguide. Such degradation could be interpreted as the fieldscreening effect in the highly structurally disordered parts of the proton exchanged regions below the surface. Besides, it was recently reported [6] that this subsurface disorder provides some marked level of propagation loss related mainly to guided mode scattering within inhomogeneous near-surface part of the waveguide, and also, significant loss of optical non- linearity [7]. It has been established [5] that the loss of water during annealing is a major contributor to the formation of crystalline disorder and, therefore, annealing under a high pressure of water vapor prevents such a disorder to appear. However, using this method only a partial suppression of the subsurface disorder and the related degradation of waveguide properties were obtained [8]. In this paper we show that, using the soft proton exchange (SPE) process [9]-[11] known as allowing the fabrication of low loss and highly efficient nonlinear components, we obtain waveguides in the $\alpha$-phase $[12,13]$ and free from any water loss [6, 8, 14], and therefore, we avoid completely these undesirable effects.

\section{EXPERIMENT}

We started by fabricating a set of planar waveguides on $\mathrm{Z}$-cut $\mathrm{LiNbO}_{3}$. The study of these test samples allowed us to determine the fabrication parameters to use in order to get a low-loss single mode channel waveguide, operating at a chosen wavelength. We choose to optimize our procedure to operate between 1530 and $1560 \mathrm{~nm}$, as the fabrication of EO modulators, operating in the third telecommunication window, has already been developed. The fabrication parameters were chosen in order to obtain a waveguide composed quasi 


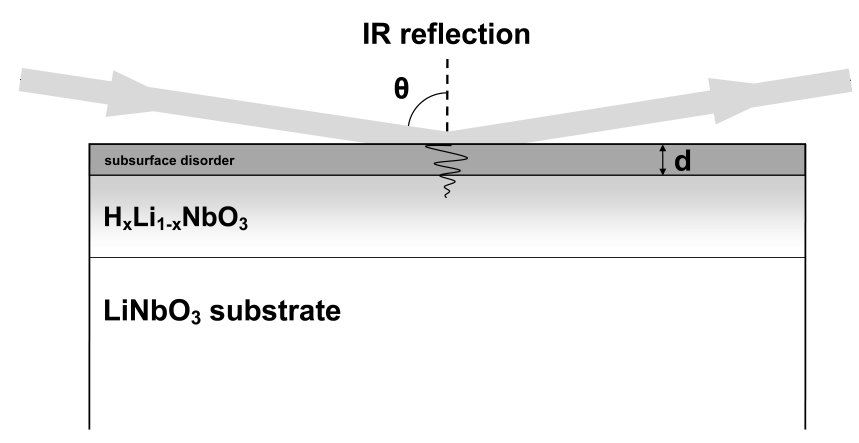

FIG. 1 Schematic draw of subsurface layer study with specular IR reflection. $d$ is thickness of the subsurface disordered part of a proton-exchanged $\mathrm{LiNbO}_{3}$ waveguiding layer. Photometered depth, $d_{p}$, at IR reflection measurements is limited by penetration depth of IR radiation in near-surface layer at glancing incidence. Hence $d_{p} \leq 1 \mu \mathrm{m}$ that is about $d$.

exclusively of a homogeneous $\alpha$-phase layer. To determine the phase composition of the exchanged layer, we used the structural phase diagram of $\mathrm{H}_{x} \mathrm{Li}_{1-\mathrm{x}} \mathrm{NbO}_{3}$ solid solution $[8,14]$. The APE waveguides were fabricated with standard APE technique (APE1 sample was annealed in dry air at $360^{\circ} \mathrm{C}$ ) [2] and improved APE technique (APE2 sample was annealed in wet air at $\left.360^{\circ} \mathrm{C}\right)[5,8]$. The initial proton exchange of these samples was performed in pure benzoic acid at $175^{\circ} \mathrm{C}$ for 6 hours. Parameters of such an as-exchanged sample (denoted as PE) were measured in order to have reference data. Sequent annealing was made for 7 hours. SPE waveguide was fabricated in sealed ampoule $[9,10]$ and with a benzoic acid melt containing $2.9 \%$ of lithium benzoate (LB).

Subsurface disorder was studied with micro-Raman and IRreflection spectroscopy technique, as broadening of Raman phonon bands and decrease of reflectivity are well-established clear indications of a marked structural disorder in $\mathrm{LiNbO}_{3}$ [8, 15]. Raman spectroscopy was performed on planar and channel waveguides with the aid of a Jobin-Yvon LabRam HR800 spectrometer operating at excitation wavelength of $632.8 \mathrm{~nm}$. Spectra were measured in the autofocus operation mode of the spectrometer, using a $\times 100$ objective and a confocal hole of width $=50 \mu \mathrm{m}$. The IR-reflection spectra with the incidence angle $\Theta$ varying from 20 to $80^{\circ}$ (Figure 1) were measured with an IR-spectrophotometer "Bruker VERTEX 80v".

Transmission spectra in the visible and near-UV ranges were taken with a Shimadzu UV- 3101PC spectrophotometer. The optical absorption spectroscopy data in the visible and the near UV ranges can be used to evaluate the electro-optical $r_{13}$ coefficients of the proton-exchanged waveguides in $\mathrm{LiNbO}_{3}$ crystal. Indeed, the shift of fundamental absorption edge, i.e. the reduction of the band-gap energy $\Delta E_{g}$, has been related to the decrease of spontaneous polarization $P$ and, therefore, of the electro-optic coefficient $r_{13}[16,17]$. For direct measurement of the EO effect in the different $\mathrm{H}_{\mathrm{x}} \mathrm{Li}_{1-\mathrm{x}} \mathrm{NbO}_{3}$ phases, we fabricated several phase modulators consisting in a channel APE waveguide in a 0.5 -mm-thick $\mathrm{z}$-cut $\mathrm{LiNbO}_{3}$ substrate surrounded by two coplanar electrodes. The EO efficiencies of the modulators were measured using a super luminescent diode (central wavelength $\sim 1550 \mathrm{~nm}$ and a bandwidth of
$50 \mathrm{~nm})$ and a Sagnac fiber interferometer. Therefore, the phase modulator was pigtailed by PM fiber at both ends input and output.

\section{RESULTS AND DISCUSSION}

Our data give the direct evidence of high structural disorder, as the strong broadening of the Raman lines (see Figure 2) shows clearly an increase of the phonon damping (related to crystalline disorder [14]) in the PE and APE waveguides with any $\mathrm{H}_{x} \mathrm{Li}_{1-x} \mathrm{NbO}_{3}$ phase, even $\alpha$-phase with low $x$. The proton exchange degree, $x$, has been determined using its well known relations with the exchange-induced refractive index change $\Delta n^{(e)}$, determined from dark m-lines spectroscopy data and from Raman data on phonon frequencies $[8,14,18]$. The region of Raman spectra ranging from $550 \mathrm{~cm}^{-1}$ to $900 \mathrm{~cm}^{-1}$ have been studied in details (Figure 2 and Table 1), as important information on the structural disorder can be obtained from Raman data of this range [8]. In particular the $\mathrm{A}_{1}(\mathrm{LO})$-phonon mode was established to be a good marker of structural disorder [8, 20]. It has been demonstrated that the shape of the related Raman band is markedly affected by the presence of structural disorder. According to the significant difference in FHWM of this Raman band between waveguides studied, its evident that subsurface disorder in SPE waveguides fabricated at appropriate conditions, e.g. SPE sample, is much smaller than in any APE samples. The clear broadening of the $A_{1}(\mathrm{LO})$ mode peak in APE waveguides is accompanied by an increase of the signal in the extra band centered at about $660-690 \mathrm{~cm}^{-1}$. Such an extra band has been attributed to structural disorder in $\mathrm{Nb}-\mathrm{O}$ ferroelectric chains [20]. In this energy region the fitting procedure is more difficult due to the superposition of different modes. Nevertheless, the comparative study of the structural disorder in the different waveguides is possible and, thus, one can observe that for the SPE waveguides this extra band is much smaller, demonstrating a less disordered structure, if compared with APE waveguides.

Besides, Raman intensity of this band was measured focusing the laser beam at different depth in the waveguides. The surface Raman intensity of this extra band is much higher than that obtained when the laser spot is at depth greater than $1.5 \mu \mathrm{m}$ for any APE waveguide fabricated. For the SPE waveguide it is evident that the damaged surface layer is less pronounced and thinner than that of the "best" APE waveguide, as the extra band intensity at the surface is always smaller and decreases more rapidly with depth, reaching the bulk value at a depth as small as $1.0 \mu \mathrm{m}$.

This result is confirmed by the measurement of the glancing IR-reflection. In this experiment, an attenuation of the reflectivity $R$ within wavenumbers $v$ range 580 to $880 \mathrm{~cm}^{-1}$ (Figure 3) is an indication of structural disorder. The disorder factor $F=1-R_{s} / R_{0}$, with $R_{s}$ and $R_{0}$ being the IR-reflection coefficients measured at $\Theta=80^{\circ}$ in a sample with a $\mathrm{H}_{x} \mathrm{Li}_{1-\mathrm{x}} \mathrm{NbO}_{3}$ waveguide and a pure $\mathrm{LiNbO}_{3}$, respectively can be evaluated from these data $[8,15]$. According to the data on vibrational spectra of disordered solids [15], such an attenuation of $\mathrm{R}$ could be interpreted as phonon scattering 


\begin{tabular}{|c|c|c|c|c|c|c|}
\hline sample & $\begin{array}{c}\text { IR } \\
\mathrm{F} \\
\end{array}$ & $\begin{array}{c}\text { Raman } \\
\Delta v_{1 / 2}, \mathrm{~cm}^{-1}\end{array}$ & $\begin{array}{l}\text { Raman } \\
I_{L O} / I_{T O}\end{array}$ & $\begin{array}{c}\text { Raman } \\
I_{\text {extra }} / I_{T O}\end{array}$ & $\begin{array}{c}\text { UV-absorption } \\
\Delta E_{g, S}, \mathrm{eV}\end{array}$ & $r_{S}(\mathrm{loc}) / r_{0}$ \\
\hline $\mathrm{z}_{0}$, pure LN & 0 & 29.7 & 3.5 & 0 & 0 & 1 \\
\hline SPE & 0.05 & 35.3 & 3.2 & 0.14 & -0.010 & 0.93 \\
\hline APE2 & 0.082 & 40.5 & 3.1 & 0.18 & -0.013 & 0.90 \\
\hline APE1 & 0.095 & 44.1 & 3.0 & 0.24 & -0.016 & 0.88 \\
\hline PE (no annealing) & 0.298 & 37.8 & 1.5 & 2.6 & -0.119 & 0.19 \\
\hline
\end{tabular}

TABLE 1 Sample notation, $F$ is the disorder factor, $\Delta v_{1 / 2}$ is the FWHM of the Raman $\mathrm{A}_{1}$ (LO)-phonon band at $871 \mathrm{~cm}^{-1}$, $I_{L O}$ and $I_{\text {extra }}$ are the Raman intensities of the $\mathrm{A} 1$ (L0)-phonon band and extra band, $\Delta E_{g, S}$ is the band-gap shift for the ordinary polarization and $r_{S}($ loc $) / r_{0}$ is the normalized values of local electro-optic coefficient $r_{13}$.



FIG. 2 Raman spectra of the subsurface part of the different samples: PE, APE1, APE2, SPE and $z_{0}$ (notation meaning is given in Table 1 ). This samples order corresponds to spectra sequence from bottom to top at $871 \mathrm{~cm}^{-1}$ (the wavenumber of $A_{1}(L 0)$-phonon band).

effects in the highly structurally disordered parts of the proton exchanged regions. Note, that a photometered depth of near-surface area at reflectivity maximum (i.e., within range of the so-called residual rays [15]) is about $1 \mu \mathrm{m}$ for glancing incidence of IR-radiation (i.e., when reflection angle $\Theta \geq 70^{\circ}$ ), Figure 1. Indeed, the photometered depth becomes much more significant at reflection angles that are far from glancing reflection and closer to the normal incidence [12]. Our data for all the studied APE and SPE waveguides show, that a relative attenuation of $R$ (normalized to reflectivity value measured in virgin $\mathrm{LiNbO}_{3}$ at a given $\Theta$ ) tends to 0 at such a decrease of $\Theta$. Thus IR-reflection factor $F$ is a well adapted parameter to study the subsurface part of the waveguides.

It is well known that proton-exchange waveguides present a drastic decrease of the spontaneous polarization $P_{0}[8,15]$ and polarizability [7]. This variation of $P_{0}$ is related to the shift of the absorption edge $[16,17]$, i.e. the change of the band gap energy $\Delta E_{g}$ :

$$
\Delta E_{g} \approx a \Delta P_{0}
$$

with $a=-0.35 \mathrm{eVm}^{4} / \mathrm{C}^{2}$ [8]. Since linear EO effect is basically a quadratic effect biased by the $P_{0}$, we can write:

$$
r_{13, S} / r_{13,0}=\left(\eta_{0} / \eta_{S}\right)^{3} \times\left\{1+\frac{\Delta E_{g, S}}{a P_{o}^{2}}\right\}
$$

where $\eta$ is the packing density, subscripts $s$ and 0 indicate val-

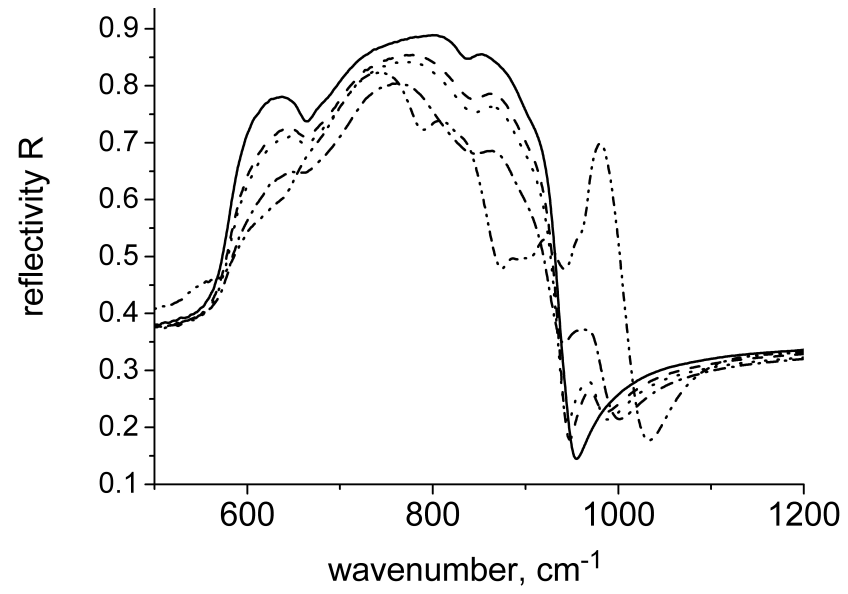

FIG. 3 IR-reflection spectra of the different samples measured at glancing incidence (incidence angle $\Theta=80^{\circ}$ ): PE, APE1, APE2, SPE and $z_{0}$. This samples order corresponds to spectra sequence from bottom to top at $871 \mathrm{~cm}^{-1}$. Incident IR light was s-polarized (i.e., it was polarized perpendicular to plane of incidence (Figure 1)).

ues for $\mathrm{H}_{\mathrm{x}} \mathrm{Li}_{1-\mathrm{x}} \mathrm{NbO}_{3}$ waveguide and virgin $\mathrm{LiNbO}_{3}$ crystals, respectively. Note, that the values of $\Delta E_{g, s}=E_{g, s}-E_{g, 0}$ have a negative sign in all the waveguides studied, as we observe a red-shift of the absorption edge in $\mathrm{H}_{\mathrm{x}} \mathrm{Li}_{1-\mathrm{x}} \mathrm{NbO}_{3}$ waveguide compared to virgin $\mathrm{LiNbO}_{3}$ (Figure 4). Band-gap energy $E_{g, s}$ can be estimated from data on the apparent fundamental absorption edge $E_{S}(m)$ in the studied sample [8] with the following equation :

$$
\begin{aligned}
E_{g, S}= & E_{S}(m) \\
& +\left\{\left(E_{g .0}-E_{S}(m)\right)^{2}+(\gamma / 2)^{2} \ln \left(h D_{0}\right) / L D_{s}\right\}^{1 / 2}
\end{aligned}
$$

where, $E_{n}(m)$ is the measured energy of the absorption edge, $h$ is the thickness of the waveguiding layer, $g$ is the damping coefficient of the electronic oscillator, $D_{0}$ is the optical density in pure $\mathrm{LiNbO}_{3}$ at $E_{S}(m), D_{s}=D_{s}(m)-D_{0}$ is the optical density of the PE layer, $D_{\mathcal{S}}(m)$ is the optical density at $E_{S}(m)$, $L$ is the sample thickness. The values of $\gamma$ are different for ordinary and extraordinary polarization: $\gamma^{(o)}=0.547 \mathrm{eV}$ and $\gamma^{(e)}=0.702 \mathrm{eV}$ [8]. By using the data on the band-gap shift, the so-called local (microscopic) values of the EO coefficient $r_{13}$ were evaluated by Eqn. 1 for all the fabricated samples, Table 1 . The results obtained demonstrate a significant dependence of the microscopic EO susceptibility on the phase composition of the APE and SPE waveguides. To determine phase composition of SPE and APE waveguides, we use Raman and IR-reflection spectroscopy, as each $\mathrm{H}_{\mathrm{x}} \mathrm{Li}_{1-\mathrm{x}} \mathrm{NbO}_{3}$ phase has a specific phonon spectrum $[6,8,19]$. 


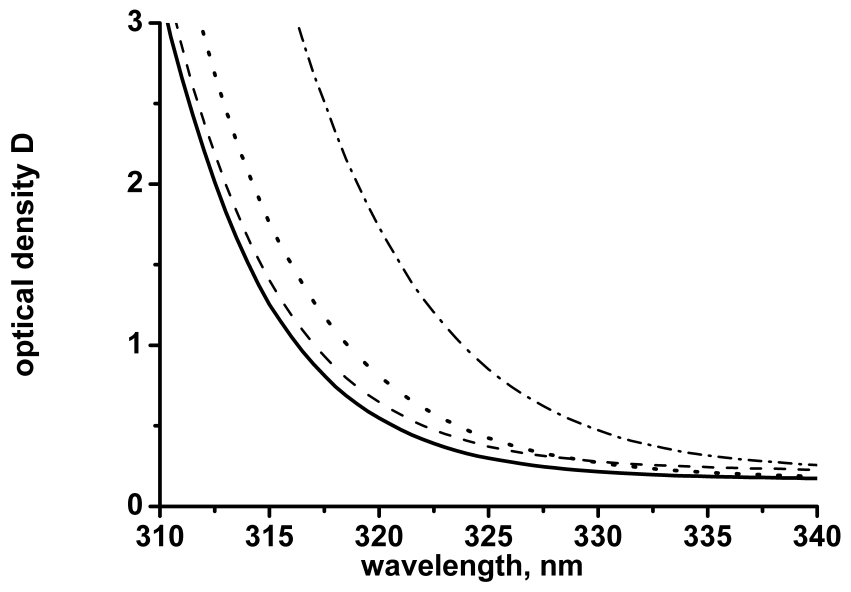

FIG. 4 Optical absorption spectra of the different samples: $z_{0}$, SPE, APE1 and PE. This samples order corresponds to spectra sequence from left to right side at $D=2$. Absorption spectrum of $z$-cut wafer of a pure congruent $\mathrm{LiNbO}_{3}$ crystal (sample $z_{0}$ ) is given as reference data for evaluation of band-gap energy changes.

\begin{tabular}{|c|c|c|c|c|c|}
\hline sample & APE1 [1] & APE1 our & APE2 & SPE & PE $^{* *}$ \\
\hline$r_{13}^{\prime}$ (loc) & $\sim 0.85^{*}$ & 0.88 & 0.90 & 0.93 & 0.19 \\
\hline$r_{13}^{\prime}$ (eff) & 0.69 & 0.80 & 0.85 & 0.90 & $\leq 0.1$ \\
\hline$F$ & $\sim 0.12^{*}$ & 0.095 & 0.082 & 0.05 & 0.22 \\
\hline
\end{tabular}

*- values evaluated by us for test samples fabricated by technique reported in Ref.[1] **- values for a sample proton-exchanged in pure benzoic acid at $175^{\circ} \mathrm{C}$ for 6 hours.

TABLE 2 The normalized values of local and effective electro-optic coefficient $r_{13}^{\prime}=r_{13, S} / r_{13,0}$, where $r_{13, S}$ and $r_{13,0}$ are values for a sample $S$ studied and pure $\mathrm{LiNbO}_{3}$. Ratio between $r_{13}^{\prime}$ (eff) and $r_{13}^{\prime}(\mathrm{loc})$ correlates with actual value of disorder factor $F$.

For direct measurement of $\mathrm{EO}$ effect in the different $\mathrm{H}_{\mathrm{x}} \mathrm{Li}_{1-\mathrm{x}} \mathrm{NbO}_{3}$ waveguides, we fabricated the EO phase modulators based on channel APE waveguides in a 0.5-mmthick x-cut $\mathrm{LiNbO}_{3}$ substrate. The electrode structure consists in two electrodes coplanar with the waveguide. In our case of Z-cut, Y-propagating geometry of single-mode APE and SPE $\mathrm{LiNbO}_{3}$ waveguides, the effective value of electro-optic coefficient $r_{13}$ (eff) is inversely proportional to the half-wave voltage $V_{\pi}$ of a phase modulator :

$$
r_{13}(\mathrm{eff})=\frac{\lambda G}{n_{e}^{3} V_{\pi} O L}
$$

where $L$ is the length of the electrodes in each arm, $O$ is the overlap factor between the field created by the electrodes and the optical mode, $\lambda$ is the wavelength of the guided light (central wavelength of SLD used is $1534 \mathrm{~nm}), n_{e}$ is the effective refractive index of the $\mathrm{TM}_{0}$ mode and $G$ is the gap width between electrodes. The local values of $r_{13}$ evaluated from $\Delta E_{g, s}$ are larger than the effective values derived from the direct measurement of the EO efficiency of the phase modulators (Table 2) that can be related to the field-screening effect in a disordered subsurface part of the waveguide. This degradation of the effective values of the EO coefficient $r_{13}$ (eff) correlates with the IR-reflection data on structural disorder at subsurface area of waveguides:

$$
r_{13}(\mathrm{eff}) \sim r_{13}(\mathrm{loc})[1-\mathrm{CF}]
$$

Broadening of Raman band correlates with the variation of the disorder factor $F$, and, therefore, Raman data may be used



FIG. 5 Correlation between disorder factor $F$ determined from IR-reflection data and broadening of the Raman band at $871 \mathrm{~cm}^{-1} \cdot v_{1 / 2}$ is the change of FWHM of this Raman band measured in subsurface area with confocal micro-Raman spectroscopy.

for the evaluation of the disorder-induced reduction of the effective electro-optic coefficient. To reach easy possibility of such evaluation, we obtain experimental calibration curve for $F$ vs $v_{1 / 2}$ (its a FWHM of $\mathrm{A}_{1}(\mathrm{LO})$-phonon band), Figure 5 . It should be noted, that micro-Raman spectroscopy can be used for study of channel waveguides in contrast to IR-reflection spectroscopy that requires the preparation of planar waveguides. Considering that electro-optic susceptibility is directly related to Raman scattering cross-section [8, 21], micro-Raman measurements were performed to detect any change in the Raman active phonon modes. It is also known that the contribution of the $\mathrm{Nb}-\mathrm{O}$ bond to the linear and nonlinear susceptibility is larger than the contributions of the Li-O bond. Mode at $870 \mathrm{~cm}^{-1}$, associated with the $\mathrm{Nb}-\mathrm{O}_{6}$ octahedron belongs to the dominant contributors to the EO effect $[19,20]$. Therefore, a simple correlation between the related Raman intensities and the electro-optic coefficients can be found. In fact, recent findings show that attenuation of Raman intensity $\left(I_{L O}\right)$ of the LO-phonon band at $871 \mathrm{~cm}^{-1}$ are related to the decrease of the local electro-optic response [8, 21]. Moreover, measurement of Raman intensities of certain phonon bands allows for rough estimation of local values of electro-optic coefficients in waveguides in different areas of any protonexchanged $\mathrm{LiNbO}_{3}$ channel waveguide.

\section{CONCLUSION}

In this paper we first describe new simple methods to estimate the disorder and the reduction of the EO coefficients induced in $\mathrm{LiNbO}_{3}$ by the different kind of known proton exchange processes. The experimental characterizations of a set of planar waveguides fabricated by different PE techniques show that the SPE process allows preparing devices with the smaller degradation of the crystalline quality of the subsurface layer and the smaller reduction of the effective electrooptic coefficient. This is confirmed by the characterization of a set of phase modulators prepared by the standard and the improved APE techniques as well as by SPE. SPE devices exhibit the higher electro-optical efficiency and the lower prop- 
agation losses. We can also expect that the SPE modulators will present a low drift in the DC bias, as subsurface disorder was previously identified as a key source of such a drift.

\section{ACKN OWLED GMENTS}

O.G. Sevostyanov and I.M. Chrikova acknowledge the Ministry of Education and Science of Russia (Project No 3.392.2014K).

\section{References}

[1] M. Rottschalk, A. Rasch, and W. Karthe, "Electrooptic behaviour of proton-exchanged $\mathrm{LiNbO}_{3}$ optical waveguides," J. Opt. Commun. 9, 19-23 (1988).

[2] A. Loni, R. M. De La Rue, and J. M. Winfield, "Very low-loss protonexchanged waveguides with a substantially restored electro-optic effect," in Proceedings of Topical Meeting on Integrated and Guided Wave Optics, Paper MD3-1, (TX, Houston, 1989).

[3] T. T. Lay, Y. Kondo, and Y. Fujii, “Effect of annealing on electrooptic constant of undoped and the MgO-doped lithium niobate optical waveguides," IEICE T. Electron 74, 3870-3872 (1991).

[4] R. Narayan, "Electrooptic coefficient variation in proton-exchanged and annealed lithium niobate samples," IEEE J. Sel. Top. Quant. 3, 796-807 (1997).

[5] H. G. Muller, A. D. Stapleton, B. J. Foran, G. Radhakrishnan, H. I. Kim, P. M. Adams, and R. A. Lipeles, "Reduction of lattice defects in proton-exchanged lithium niobate waveguides," J. Appl. Phys. 110, 033539 (2011).

[6] S. M. Kostritskii, Y. N. Korkishko, V. A. Fedorov, A. N. Alkaev, V. Kritzak, P. Moretti, and S. Tascu, "Leakage of a guided mode caused by static and light-induced inhomogeneities in channel HTPE-LiNb03 waveguides," Proc. SPIE. 4944, 346-352 (2003).

[7] F. Laurell, M. G. Roelofs, and H. Hsiung "Loss of optical nonlinearity in proton-exchanged $\mathrm{LiNbO}_{3}$ waveguides", Appl. Phys. Lett. 60, 301-303 (1992).

[8] S. M. Kostritskii, S. V. Rodnov, Y. N. Korkishko, V. A. Fedorov, and 0 . G. Sevostyanov, "Electro-optical properties of different $\mathrm{H}_{\mathrm{x}} \mathrm{Li}_{1-\mathrm{x}} \mathrm{NbO}_{3}$ phases in proton-exchanged $\mathrm{LiNbO}_{3}$ waveguides," Ferroelectrics 440, 47-56 (2012).
[9] L. Chanvillard, P. Aschieri, P. Baldi, D. B. Ostrowsky, M. De Micheli, and L. Huang, "Soft Proton Exchange on PPLN: a simple waveguide fabrication process for highly efficient nonlinear interactions," Appl. Phys. Lett. 76, 1089 (2000).

[10] D. Castaldini, P. Bassi, S. Tascu, P. Aschieri, M.P. De Micheli, and P. Baldi, "Soft proton exchange tapers for low-insertion loss LiNb03 devices," J. Lightwave Technol. 25, 1588-1593 (2007).

[11] M. P. De Micheli, "Fabrication and Characterization of Proton Exchanged Waveguides in Periodically Poled Congruent Lithium Niobate," Ferroelectrics 340, 49-62, (2006).

[12] M. De Micheli, D. Ostrowsky, J. Barety, C. Canali, A. Carnera, G. Mazzi, and M. Papuchon, "Crystalline and optical quality of proton exchanged waveguides," J. Lightwave Technol. 4, 743-745 (1986).

[13] Y. N. Korkishko, V. A. Fedorov, M. De Micheli, K. El Hadi, P. Baldi, and A. Leycuras. "Relationships between structural and optical properties of proton-exchanged waveguides on Z-cut lithium niobate," Appl. Optics 35, 7056-7060 (1996).

[14] Y. N. Korkishko, V. A. Fedorov, E. A. Baranov, M. V. Proyaeva, T. V. Morozova, F. Caccavale, F. Segato, et al., "Characterization of $\alpha$-phase soft proton-exchanged $\mathrm{LiNbO}_{3}$ optical waveguides", J. Opt. Soc. Am. A. 18, 1186-1191 (2001).

[15] A. S. Barker, and A. J. Sievers, "Optical studies of the vibrational properties of disordered solids", Rev. Mod. Phys. 47, 1-179 (1975).

[16] H. Ahlfeldt, J. Webjorn, P. A. Thomas, and S. J. Teat, "Structural and optical properties of annealed proton-exchanged waveguides in z-cut LiTa03," J. Appl. Phys. 77, 4467-4476 (1995).

[17] S. H. Wemple, M. Di Domenico, and I. Camlibel, "Relationship between linear and quadratic electro-optic coefficients in LiNb03," Appl. Phys. Lett. 12, 209-212 (1968).

[18] M. Di Domenico, and S. H. Wemple, "Oxygen-octahedra ferroelectrics. I. Theory of electro-optical and nonlinear optical effects," J. Appl. Phys. 40, 720-732 (1969).

[19] M. Kuneva, and S. Tonchev, "Spectroscopy of optical waveguiding layers", Bulg. Chem. Commun. 43, 276-287 (2011).

[20] G. R. Paz-Pujalt, D. D. Tuschel, G. Braunstein, T. Blanton, S. Tong Lee, and L. M. Salter, "Characterization of proton-exchange lithium-niobate wave-guides," J. Appl. Phys. 76, 3981-3987 (1994).

[21] I. P. Kaminow, and W. D. Johnston, "Quantitative determination of sources of electro-optic effect in $\mathrm{LiNbO}_{3}$ and $\mathrm{LiTaO}_{3}$," Phys. Rev. 160, 519-522 (1967). 